\title{
AN ATYPICAL CASE OF CIRRHOSIS
}

\section{SAKETH RAMINENI ${ }^{1}$, PADMA $V^{2}$, KAVI M G ${ }^{3}$, SANDHYA PC ${ }^{4}$, MURUGARAJ R ${ }^{5}$, ABHILASH NAIR ${ }^{5}$, KANNAN MEERA DEVI ${ }^{5}$ AND SARATH BHASKAR ${ }^{5}$}

1: Junior Resident, Department of Medicine, Sree Balaji Medical College and Hospital, Chrompet, Chennai-600044

2: Professor, Department of Medicine, Sree Balaji Medical College and Hospital, Chrompet, Chennai600044

3: Assistant Professor, Department of Medicine, Sree Balaji Medical College and Hospital, Chrompet, Chennai-600044

4: Senior Resident, Department of Medicine, Sree Balaji Medical College and Hospital, Chrompet, Chennai-600044

5: Resident, Department of Medicine, Sree Balaji Medical College and Hospital, Chrompet, Chennai600044

*Corresponding Author: Dr. Saketh Ramineni: rsaketh1992@gmail.com

Received 26 ${ }^{\text {th }}$ March 2021; Revised 27 ${ }^{\text {th }}$ April 2021; Accepted 24 ${ }^{\text {th }}$ May 2021; Available online $1^{\text {st }}$ Dec. 2021

\section{https://doi.org/10.31032/IJBPAS/2021/10.12.5792}

ABSTRACT

The term cirrhosis was first used in the year 1826. Cirrhosis is defined as a process which involves the conversion of normal liver into structurally abnormal nodules. The progression into cirrhosis from an injury may take weeks to years. Common causes which lead to this condition are Hepatitis C, Alcohol, Hepatitis B and cryptogenic causes. Here we present a case of liver cirrhosis which was caused by pericarditis.

Keywords: Pericardial calcification, Cardiac catherterization, Liver cirrhosis

\section{INTRODUCTION}

Constrictive pericarditis is due to chronic inflammation of pericardium which leads to decreased ventricular function [1]. It is important to evaluate any cardiac related etiology in liver dysfuntion because such conditions can be reversed with prompt treatment $[2,3]$.

Below we report a case of 40 year old female where cirrhosis was incidentally found. Pericardial calcifications were 
shown on imaging which was diagnosed as constrictive pericarditis

\section{CASE REPORT}

A 40 year old female who is a known case of sarcoidosis was found to have a nodular liver in imaging on general check up. There was no yellowish discoloration of eyes, Hematemesis and no confusion. There was no history of alcohol use. Physical examination was normal. Liver function tests revealed elevated alkaline phosphatase and PT was prolonged, mild thrombocytopenia was noted. No abnormalities were noted in hepatitis serologies. Liver biopsy confirmed cirrhosis. We took an opinion from the cardiologist after sinusoidal dilatation was seen in liver biopsy. Englarged IVC was seen in echo cardiogram. A coronary angiogram was done. Coronary arteries was normal. On Right heart catheterisation an RAP of $19 \mathrm{~mm} \mathrm{Hg}$, PAP of $33 / 15 \mathrm{~mm}$ of Hg. PAWP of $19 \mathrm{~mm}$ of $\mathrm{Hg}$. Cardiac output was $5.16 \mathrm{~L} / \mathrm{min}$ Pulmonary medicine opinion was taken after shortness of breath developed. Mild restriction was noted in pulmonary function tests. CT pulmonary angiogram showed pericardial calcification. She developed one episode of SVT, Catheterization was repeated again. RAP is $24 \mathrm{~mm}$ of $\mathrm{Hg}$, PAP is $51 / 25 \mathrm{~mm}$ of $\mathrm{hg}$, PAWP is $31 \mathrm{~mm}$ of hg. Cardiac output was 4.02 L/min. Pressures of both left and right heart were recorded simultaneously. This lead to the diagnosis of constrictive pericarditis. We came to a conclusion that constrictive pericarditis was caused by sarcoidosis.

\section{DISCUSSION}

A complete work up of cirrhosis is needed when common causes cannot be linked to the case. Until frank cirrhosis has developed serum albumin will be in normal range. Elevation of serum albumin- ascetic gradient typical of portal hypertension is noted in ascetic fluid analysis [4]. In this case there was only alkaline phosphate elevation with PT elevation, finding nodular liver was incidental. Sinusoidal dilation on biopsy helped us to think of a cardiac etiological factor. Ascites is generally seen in patients with constrictive pericarditis, however no such finding was seen.

Development of dyspnea in liver dysfuntion is because of hepatopulmonary syndrome, cardiomyopathy and portopulmonary hypertension. Constrictive pericarditis should be taken into account when there is no explained cause of cirrhosis [5]. After finding pericardial calcification one should think about constrictive pericarditis. One study interpreted that low cost imaging in such condition helps is timely diagnosis [6]. Restrictive cardiomyopathy is a common differential in patients with sarcoidosis. So cardiac catheterization is mandatory for 
differentiating these two conditions.

Respiratory variation, atrial pressures and end diastolic pressures should be recorded by simultaneous right and left cardiac catheterisations [7]. Timely diagnosis and treatment of underlying cardiac disease will help in prevention of hepatocellular carcinoma, further it helps in reversing the liver condition and prevents cirrhosis.

\section{CONCLUSION}

Gastroenterologists need to consider a full cardiac work up in unexplained causes of liver cirrhosis. Presence of pericardial calcification in a patient with liver cirrhosis should point out the etiology towards cardiac cirrhosis and constrictive pericarditis. Cardiac catheterization and cardiac CTs will provide a better insight into the condiyion. Reffering the patient to a cardio thoracic surgeon for pericardiectomy will help in prevention of cirrhosis.

\section{REFERENCES}

[1] B. Maisch, P. M. Seferovic, A. D. Risti c et al., Guidelines on the diagnosis and management of pericardial diseases executive Case Reports in Gastrointestinal Medicine 3 summary; The Task force on the diagnosis and management of pericardial diseases of the European society of cardiology. European Heart Journal., 2004; 25: 7; 587-610.

[2] F. Heureux, L. Frankart, B. Marchandise, M. Buche, J. P. Martinet, and J. Donckier. Recurrent ascites: two case reports. Acta Clinica Belgica, 1997; 52: 3: 176-181.

[3] A. Sheth and J. K. Lim. Liver disease from asymptomatic constrictive pericarditis. J Clinical Gastroenterology., 2008; 42: 8: 956-958.

[4] C. C. Giallourakis, P. M. Rosenberg, and L. S. Friedman. The liver in heart failure. Clinics in Liver Disease., 2002; 6:4:947-967.

[5] M. Kirsch and B. Fleshler. Deceptive liver histology delays diagnosis of cardiac ascites. Southern Medical Journal., 1992; 85: 11: 1151-1152.

[6] M. Toledano and A. Bhagra. Pericardial calcification in constrictive pericarditis. International Journal of Emergency Medicine., 2012; 5: 37.

[7] M. H. Khandaker, R. E. Espinosa, R. A. Nishimura et al., "Pericardial disease: diagnosis and management," Mayo Clinic Proceedings, vol. 85, no. 6 , pp. 572-593, 2010. 